\title{
Antithyroid Antibodies and Thyroid Function in Pediatric Patients with Celiac Disease
}

\author{
Derya Kalyoncu ${ }^{1}$ and Nafiye Urganci ${ }^{2}$ \\ ${ }^{1}$ Department of Pediatrics, Sisli Etfal Training and Research Hospital, 34270 Istanbul, Turkey \\ ${ }^{2}$ Division of Pediatric Gastroenterology, Sisli Etfal Training and Research Hospital, 34270 Istanbul, Turkey
}

Correspondence should be addressed to Derya Kalyoncu; deryakaly@yahoo.com

Received 10 September 2014; Accepted 11 February 2015

Academic Editor: Jack R. Wall

Copyright (C) 2015 D. Kalyoncu and N. Urganci. This is an open access article distributed under the Creative Commons Attribution License, which permits unrestricted use, distribution, and reproduction in any medium, provided the original work is properly cited.

\begin{abstract}
Objective. Aim of the study was to determine the prevalence of autoimmune thyroid disease, persistence of antithyroid antibodies, effect of gluten-free diet, and long-term outcome of thyroid function in pediatric patients with celiac disease (CD). Methods. 67 patients with CD aged from 1 year to 16 years were screened for thyroid antithyroperoxidase, antithyroglobulin and anti-TSH receptor antibodies, serum free triiodothyronine, free thyroxine, and thyroid-stimulating hormone (TSH) at diagnosis and during follow-up. Results. None of the patients had antithyroid antibodies at diagnosis. Antithyroid antibodies became positive in $16.4 \%$ of the patients (11/67) 2 to 3 years after the diagnosis of CD. Clinical hypothyroidism was observed only in 3 of 11 CD patients with positive antithyroid antibodies $(27.2 \%)$. The antithyroid antibodies positive and negative patients did not differ significantly according to compliance to GFD $(P>0.05)$. A statistically significant difference was observed only in age, in which the patients with positive antithyroid antibodies were younger than the patients with negative antithyroid antibodies $(P=0.004)$. None of the patients had any change in their thyroid function and antibody profile during their follow-up. Conclusion. Antithyroid antibodies were detected in younger pediatric patients with $\mathrm{CD}$ and the prevalence of antithyroid antibodies did not correlate with the duration of gluten intake.
\end{abstract}

\section{Introduction}

Celiac disease (CD) is an immune-mediated disease triggered by an environmental agent, gluten, in genetically predisposed individuals, characterized by villous atrophy of the proximal small intestine and malabsorption. Gluten induces the production of specific autoantibodies directed against tissue transglutaminase not only in the small intestine but also in other tissues.

The association between $\mathrm{CD}$ and other autoimmune diseases such as type 1 diabetes mellitus, autoimmune thyroiditis, and other endocrine diseases has been reported in many studies in both children and adults [1-19]. It has been suggested that prolonged exposure to gluten in $\mathrm{CD}$ may promote the development of other autoimmune diseases by causing production of autoantibodies [2]. This coexistence is thought to be partly due to HLA-DQ2 and DQ8 haplotypes which are overrepresented in many autoimmune diseases $[20,21]$ and the inheritance of these haplotypes
[19]. Besides similar HLA haplotypes, association with the gene encoding cytotoxic T-lymphocyte-associated antigen4 (CTLA-4), a candidate gene for conferring susceptibility to thyroid autoimmunity, also has been reported $[19,22-$ 24]. Among autoimmune disorders, thyroid abnormalities were reported to be $26.2 \%$ [13], 37.6\% [12], and $41.1 \%$ [14] in pediatric patients with $\mathrm{CD}$.

This study was carried out to determine the prevalence of autoimmune thyroid disease, the relationship between antithyroid antibodies and gluten-free diet (GFD), persistence of antithyroid antibodies during follow-up, and longterm outcome of thyroid function in children with $\mathrm{CD}$.

\section{Materials and Methods}

Sixty-seven children with CD diagnosed and followed up between 1999 and 2011 at Division of Pediatric Gastroenterology of Sisli Etfal Training and Research Hospital (Istanbul, Turkey) were evaluated prospectively. The diagnosis of $\mathrm{CD}$ 
was based on ESPGHAN criteria [25]. The histopathological changes of small intestinal biopsies were graded according to a modified Marsh classification [26]. Informed consents were obtained from all of the parents before the procedures. During follow-up, dietary compliance to GFD was evaluated on every visit by the same physician and celiac disease activity was monitored by measurement of antibodies against transglutaminase and endomysium. A family history of autoimmune diseases was determined among first-degree relatives of all patients.

Baseline thyroid antithyroperoxidase (TPOAb), antithyroglobulin (TGAb) and anti-TSH receptor (TRAb) antibodies, serum free triiodothyronine (FT3), free thyroxine (FT4), and thyroid-stimulating hormone (TSH) were examined in all patients. FT3, FT4, and TSH were detected by enhanced chemiluminescence immunoassay (ECLIA) using commercial kits (normal values, FT4: $0.86-1.78 \mathrm{ng} / \mathrm{dL}$; FT3: $3.2-6.8 \mathrm{pmol} / \mathrm{L}$; TSH: 0.7-5.97 mU/L). Serum antithyroid antibody titers were detected using chemiluminescent immunoassay (CLIA) and radioimmunoassay (RIA). Antithyroid antibodies and thyroid function tests were measured regularly once every year during follow-up. In children with increased antibody titers, thyroid ultrasonography was performed.

The diagnosis of autoimmune thyroid disease was based on positivity of antithyroid antibodies and diffuse or irregular hypoechogenicity of the thyroid gland on ultrasound examination. Euthyroidism was defined as normal TSH and FT4 levels. Clinical hypothyroidism was defined as an increase in TSH serum concentration and a significant decrease in FT4 level. Subclinical hypothyroidism was recognised by TSH elevation with normal concentrations of circulating thyroid hormones (FT4 and FT3). Hyperthyroidism was defined as suppressed, usually undetectable serum TSH concentration and increased FT4 and FT3. Subclinical hyperthyroidism was noted by decreased TSH serum levels and normal FT4.

2.1. Statistical Analysis. Statistical analyses were performed using SPSS 11.0 software (SPSS Inc., Chicago, IL, USA). Results were expressed as means \pm SD for quantitative variables and proportions for categorical variables. The analysis was conducted using Fisher's exact test, chi-square test, and ANOVA to analyze qualitative variables. $P$ values of $<0.05$ were considered statistically significant.

\section{Results}

The age of patients ranged from 1 year to 16 years (mean $6.77 \pm 4.64$ ), and male : female ratio was 0.59 . No patient had a family history of autoimmune diseases. $16.4 \%$ of the patients $(11 / 67)$ had antithyroid antibodies which became positive 2 to 3 years after the diagnosis of $\mathrm{CD}$. There was no serologic or clinical sign of another autoimmune disease in patients with $\mathrm{CD}$ except for two patients with type 1 diabetes mellitus. These two patients had negative antithyroid antibodies. In two patients, autoimmune disease (type 1 diabetes mellitus) was diagnosed before $\mathrm{CD}$ while all of the autoimmune thyroid disease diagnoses were subsequent to the diagnosis of $\mathrm{CD}$.
TABLE 1: Characteristics of pediatric patients with celiac disease.

\begin{tabular}{|c|c|c|c|}
\hline CD patients & $\begin{array}{c}\text { Thyroid } \\
\text { antibodies } \\
(+) \\
(n=11)\end{array}$ & $\begin{array}{c}\text { Thyroid } \\
\text { antibodies } \\
(-) \\
(n=56) \\
\end{array}$ & $P$ \\
\hline Age at diagnosis (year) & $3.22 \pm 2.21$ & $7.47 \pm 4.69$ & 0.004 \\
\hline Gender (male/female) & $0.57: 1(4 / 7)$ & $0.6: 1(21 / 35)$ & 1.00 \\
\hline \multicolumn{4}{|l|}{ Weight } \\
\hline$<3$ rd percentile & $6(54.5 \%)$ & $29(51.7 \%)$ & 1.00 \\
\hline$>3$ rd percentile & $5(45.4 \%)$ & $27(48.2 \%)$ & 0.82 \\
\hline \multicolumn{4}{|l|}{ Height } \\
\hline$<3$ rd percentile & $6(54.5 \%)$ & $27(48.2 \%)$ & 0.75 \\
\hline$>3$ rd percentile & $5(45.4 \%)$ & $29(51.7 \%)$ & 0.70 \\
\hline \multicolumn{4}{|l|}{ Clinical presentation } \\
\hline Typical & $9(81.8 \%)$ & $32(57.1 \%)$ & 0.18 \\
\hline Atypical & $2(18.1 \%)$ & $24(42.8 \%)$ & 0.12 \\
\hline \multicolumn{4}{|l|}{ Compliance to GFD } \\
\hline Compliant & $7(63.6 \%)$ & $30(53.5 \%)$ & 0.74 \\
\hline Noncompliant & $4(36.3 \%)$ & $26(46.4 \%)$ & 0.54 \\
\hline Duration of follow-up & $8.05 \pm 3.6$ & $6.7 \pm 4.1$ & 0.31 \\
\hline \multicolumn{4}{|l|}{ Laboratory findings } \\
\hline TSH (mU/L) & $2.92 \pm 1.56$ & $2.99 \pm 1.73$ & 0.95 \\
\hline FT4 (ng/dL) & $1.10 \pm 0.31$ & $1.37 \pm 1.39$ & 0.58 \\
\hline FT3 (pmol/L) & $3.28 \pm 0.98$ & $3.25 \pm 0.45$ & 0.61 \\
\hline
\end{tabular}

GFD: gluten-free diet; TSH: thyrotropin (thyroid-stimulating hormone); FT4: free thyroxine; FT3: free triiodothyronine.

$P<0.05$ is statistically significant.

The baseline demographic and clinical characteristics of the cases are shown in Table 1.

When compared with antithyroid antibodies negative patients, the patients with positive antithyroid antibodies were younger at diagnosis and the difference was statistically significant $(P=0.004)$. The antithyroid antibodies positive and negative patients did not differ significantly in gender, weight, height, clinical presentation, and histological type according to the modified Marsh criteria and compliance to GFD $(P>0.05)$ (Table 1).

Clinical hypothyroidism was observed in 3 of those 11 CD patients with positive antithyroid antibodies (27.2\%) but none of the patients with negative antithyroid antibodies. Hyperthyroidism was diagnosed in none of the patients. All of the patients except 3 with hypothyroidism had normal thyroid function (euthyroidism) at diagnosis and none had any variation in their thyroid function and antibody profile during their follow-up. All of 3 patients with hypothyroidism were compliant with GFD. Ultrasonography showed abnormal thyroid pattern characterized by diffuse hypoechogenicity in 3 patients with hypothyroidism and normoechoic sonographic pattern in other patients.

Of 11 patients with persistently positive antithyroid antibody titers, $8(72.7 \%)$ remained consistently euthyroid during the follow-up and subclinical hypothyroidism was detected 
in none of them. Three patients with clinical hypothyroidism became euthyroid with levothyroxine therapy given.

\section{Discussion}

The association between CD and other autoimmune disorders such as type 1 diabetes mellitus, autoimmune thyroid disease, and other endocrine diseases is well established in many studies [1-19]. Early identification of autoimmune disorders in patients with $\mathrm{CD}$ is important since it may be useful in the control of autoimmune disease itself, as well as in the prevention of long-term complications of CD.

An increased prevalence of antithyroid antibodies has been reported in patients with CD [12-14]. 16.4\% of our patients had antithyroid antibodies in this study, similarly as reported in previous studies $[8,11,16,27]$ and lower than that obtained by Forchielli et al. [12], Ansaldi et al. [13], and Kowalska et al. [14].

Although some authors disagree [28, 29], it has been reported that the prevalence of autoimmune disorders in CD increased with increasing age at diagnosis [1, 15], which means late diagnosis of CD causes longer exposure to gluten and higher incidence of autoimmune diseases. Oderda et al. [16] reported that untreated children with antithyroid antibodies at diagnosis were significantly older, suggesting that the duration of gluten exposure may be another important risk factor for the development of autoimmunity. In contrast with these studies, CD patients with positive antithyroid antibodies were significantly younger than the patients with negative antithyroid antibodies in our study (mean age, 3.22 \pm 2.21 versus $7.47 \pm 4.69$ years, resp., $P=0.004)$.

Cosnes et al. [30] demonstrated that CD patients who were diagnosed earlier in life and had family history of autoimmunity were most at risk for autoimmune disorders. In particular, the first-degree relatives of CD patients have an increased risk of autoimmune diseases, most likely connected with unrecognized subclinical or silent forms of CD [31, 32]. A family history of autoimmune diseases was determined in none of our patients. It has been suggested that GFD was not sufficient to suppress thyroid autoimmunity when it has already started and early diagnosis of CD and an early gluten withdrawal may be preventive for thyroiditis [16]. CD patients with antithyroid antibodies were diagnosed in earlier ages than the patients with negative antithyroid antibodies in our study. Antithyroid antibodies became positive in 11 patients 2 to 3 years after the diagnosis of CD while $63.6 \%$ of them were compliant with GFD.

At initial phase, signs and symptoms of thyroid disease are usually absent and TSH may be normal and anti-TPO antibodies may be positive [31]. Because there is possibility of worsening of thyroid function over time, early recognition of thyroid dysfunction is necessary to prevent the negative effects of hypothyroidism on growth, metabolic function, and celiac disease. As the disease progresses, the immune response to the target cell destroys the endocrine gland, leading to hypofunction. Thus, subclinical hypothyroidism (TSH elevation with a normal FT4) and then clinical hypothyroidism (increase in TSH serum concentration and decrease in FT4 level) appear [31]. It has been suggested that long-term follow-up of euthyroid patients with positive autoimmune thyroid serology would be advisable to establish whether antithyroid antibodies could demonstrate a higher propensity for thyroid involvement [13]. The antithyroid antibodies were positive in $12.5 \%(8 / 64)$ of our euthyroid patients with CD and no change in their thyroid function was observed during their follow-up.

Although some of the previous studies have reported that duration of gluten exposure in CD does not correlate with the risk for autoimmune disease and that gluten withdrawal did not protect from autoimmune disease $[8,13,29]$, several reports suggested that adherence to GFD was associated with a decreased risk of subsequent autoimmune disease and antithyroid antibodies tend to disappear during GFD $[1,11,30]$. Guariso et al. [17] have concluded that GFD seems to produce a favourable effect on the previously present clinical autoimmune disease and to prevent the development of new clinical autoimmune disease but does not affect the onset of potential autoimmunity. Our results showed that there was no significant correlation between compliance to GFD and autoimmune thyroid disease and thyroid dysfunction. 63.6\% (7/11) of the CD patients with positive antithyroid antibodies and all of the patients with hypothyroidism (3/3) were compliant with GFD; thus gluten withdrawal did not protect them. Antithyroid antibodies persisted despite GFD during follow-up. 44.7\% (30/67) of our patients were noncompliant with GFD and only 4 of them developed antithyroid antibodies.

The correlation between the degree of histological changes and the appearance of autoantibodies could not be compared in this study, because, according to the modified Marsh criteria, the disease was severe (IIIb/IIIc) in all of the patients enrolled in this study.

In conclusion, we only observed significant difference between presence of antithyroid antibodies and younger age in patients with $\mathrm{CD}$. The prevalence of antithyroid antibodies did not correlate with the duration of gluten intake and compliance to GFD in this study. Thyroid function should be assessed in all children with CD at diagnosis and during follow-up. Further, larger, prospective studies with longer follow-up are needed to clarify the clinical significance of antithyroid antibodies in pediatric patients with $C D$ and the effect of GFD and other factors on the development of autoimmune thyroid disease.

\section{Conflict of Interests}

The authors declare that there is no conflict of interests regarding the publication of this paper.

\section{References}

[1] V. Toscano, F. G. Conti, E. Anastasi et al., "Importance of gluten in the induction of endocrine autoantibodies and organ dysfunction in adolescent celiac patients," The American Journal of Gastroenterology, vol. 95, no. 7, pp. 1742-1748, 2000.

[2] A. Ventura, G. Magazù, T. Gerarduzzi et al., "Coeliac disease and the risk of autoimmune disorders," Gut, vol. 51, no. 6, pp. 897-898, 2002. 
[3] E. Schober, B. Bittmann, G. Granditsch et al., "Screening by antiendomysium antibody for celiac disease in diabetic children and adolescents in Austria," Journal of Pediatric Gastroenterology and Nutrition, vol. 30, no. 4, pp. 391-396, 2000.

[4] T. Saukkonen, E. Savilahti, H. Reijonen, J. Ilonen, E. Tuomilehto-Wolf, and H. K. Akerblom, "Coeliac disease: frequent occurrence after clinical onset of insulin-dependent diabetes mellitus," Diabetic Medicine, vol. 13, no. 5, pp. 464-470, 1996.

[5] J. C. Vitoria, L. Castaño, I. Rica, J. R. Bilbao, A. Arrieta, and M. D. García-Masdevall, "Association of insulin-dependent diabetes mellitus and celiac disease: a study based on serologic markers," Journal of Pediatric Gastroenterology and Nutrition, vol. 27, no. 1, pp. 47-52, 1998.

[6] I. Sanchez-Albisua, J. Wolf, A. Neu, H. Geiger, I. Wäscher, and M. Stern, "Coeliac disease in children with Type 1 diabetes mellitus: the effect of the gluten-free diet," Diabetic Medicine, vol. 22, no. 8, pp. 1079-1082, 2005.

[7] C. L. Ch'ng, M. Biswas, A. Benton, M. K. Jones, and J. G. C. Kingham, "Prospective screening for coeliac disease in patients with Graves' hyperthyroidism using anti-gliadin and tissue transglutaminase antibodies," Clinical Endocrinology, vol. 62, no. 3, pp. 303-306, 2005.

[8] A. Meloni, C. Mandas, R. D. Jores, and M. Congia, "Prevalence of autoimmune thyroiditis in children with celiac disease and effect of gluten withdrawal," Journal of Pediatrics, vol. 155, no. 1, pp. 51.e1-55.el, 2009.

[9] C. Sategna-Guidetti, M. Bruno, E. Mazza et al., "Autoimmune thyroid diseases and coeliac disease," European Journal of Gastroenterology and Hepatology, vol. 10, no. 11, pp. 927-931, 1998.

[10] D. Larizza, V. Calcaterra, C. de Giacomo et al., "Celiac disease in children with autoimmune thyroid disease," Journal of Pediatrics, vol. 139, no. 5, pp. 738-740, 2001.

[11] A. Ventura, E. Neri, C. Ughi, A. Leopaldi, A. Città, and T. Not, "Gluten-dependent diabetes-related and thyroid-related autoantibodies in patients with celiac disease," Journal of Pediatrics, vol. 137, no. 2, pp. 263-265, 2000.

[12] M. Forchielli, A. Collina, L. Zannarini, and M. Capelli, "Celiac disease and thyroid abnormalities: another possible association," Journal of Pediatric Gastroenterology and Nutrition, vol. 31, supplement 2, p. S63, 2000.

[13] N. Ansaldi, T. Palmas, A. Corrias et al., "Autoimmune thyroid disease and celiac disease in children," Journal of Pediatric Gastroenterology and Nutrition, vol. 37, no. 1, pp. 63-66, 2003.

[14] E. Kowalska, K. Wasowska-Krolikowska, and E. ToporowskaKowalska, "Estimation of antithyroid antibodies occurrence in children with coeliac disease," Medical Science Monitor, vol. 6, no. 4, pp. 719-721, 2000.

[15] A. Ventura, G. Magazzu, and L. Greco, "Duration of exposure to gluten and risk for autoimmune disorders in patients with celiac disease," Gastroenterology, vol. 117, no. 2, pp. 297-303, 1999.

[16] G. Oderda, A. Rapa, A. Zavallone, L. Strigini, and G. Bona, "Thyroid autoimmunity in childhood celiac disease," Journal of Pediatric Gastroenterology and Nutrition, vol. 35, no. 5, pp. 704705, 2002.

[17] G. Guariso, S. Conte, F. Presotto et al., "Clinical, subclinical and potential autoimmune diseases in an Italian population of children with coeliac disease," Alimentary Pharmacology and Therapeutics, vol. 26, no. 10, pp. 1409-1417, 2007.
[18] P. Collin, J. Salmi, O. Hallstrom, T. Reunala, and A. Pasternack, "Autoimmune thyroid disorders and coeliac disease," European Journal of Endocrinology, vol. 130, no. 2, pp. 137-140, 1994.

[19] L. C. Chin, M. K. Jones, and J. G. C. Kingham, "Celiac disease and autoimmune thyroid disease," Clinical Medicine and Research, vol. 5, no. 3, pp. 184-192, 2007.

[20] K. Kaukinen, P. Collin, A.-H. Mykkänen, J. Partanen, M. Mäki, and J. Salmi, "Celiac disease and autoimmune endocrinologic disorders," Digestive Diseases and Sciences, vol. 44, no. 7, pp. 1428-1433, 1999.

[21] K. Badenhoop, W. Dieterich, M. Segni et al., "HLA DQ2 and/or DQ8 is associated with celiac disease-specific autoantibodies to tissue transglutaminase in families with thyroid autoimmunity," The American Journal of Gastroenterology, vol. 96, no. 5, pp. 1648-1649, 2001.

[22] A. L. King, S. J. Moodie, J. S. Fraser et al., "Coeliac disease: investigation of proposed causal variants in the CTLA4 gene region," European Journal of Immunogenetics, vol. 30, no. 6, pp. 427-432, 2003.

[23] D. A. Chistiakov and R. I. Turakulov, "CTLA-4 and its role in autoimmune thyroid disease," Journal of Molecular Endocrinology, vol. 31, no. 1, pp. 21-36, 2003.

[24] K. A. Hunt, D. P. B. McGovern, P. J. Kumar et al., "A common CTLA4 haplotype associated with coeliac disease," European Journal of Human Genetics, vol. 13, no. 4, pp. 440-444, 2005.

[25] "Revised criteria for diagnosis of coeliac disease. Report of Working Group of European Society of Paediatric Gastroenterology and Nutrition," Archives of Disease in Childhood, vol. 65, no. 8, pp. 909-911, 1990.

[26] K. Rostami, J. Kerckhaert, R. Tiemessen, B. M. E. von Blomberg, J. W. R. Meijer, and C. J. J. Mulder, "Sensitivity of antiendomysium and antigliadin antibodies in untreated celiac disease: disappointing in clinical practice," The American Journal of Gastroenterology, vol. 94, no. 4, pp. 888-894, 1999.

[27] A. Diamanti, F. Ferretti, R. Guglielmi et al., "Thyroid autoimmunity in children with coeliac disease: a prospective survey," Archives of Disease in Childhood, vol. 96, no. 11, pp. 1038-1041, 2011.

[28] M. Viljamaa, K. Kaukinen, H. Huhtala, S. Kyrönpalo, M. Rasmussen, and P. Collin, "Coeliac disease, autoimmune diseases and gluten exposure," Scandinavian Journal of Gastroenterology, vol. 40, no. 4, pp. 437-443, 2005.

[29] C. Sategna Guidetti, E. Solerio, N. Scaglione, G. Aimo, and G. Mengozzi, "Duration of gluten exposure in adult coeliac disease does not correlate with the risk for autoimmune disorders," Gut, vol. 49, no. 4, pp. 502-505, 2001.

[30] J. Cosnes, C. Cellier, S. Viola et al., "Incidence of autoimmune diseases in celiac disease: protective effect of the gluten-free diet," Clinical Gastroenterology and Hepatology, vol. 6, no. 7, pp. 753-758, 2008.

[31] P. Petaros, S. Martelossi, A. Tommasini, G. Torre, M. Caradonna, and A. Ventura, "Prevalence of autoimmune disorders in relatives of patients with celiac disease," Digestive Diseases and Sciences, vol. 47, no. 7, pp. 1427-1431, 2002.

[32] F. Cataldo and V. Marino, "Increased prevalence of autoimmune diseases in first-degree relatives of patients with celiac disease," Journal of Pediatric Gastroenterology and Nutrition, vol. 36, no. 4, pp. 470-473, 2003. 


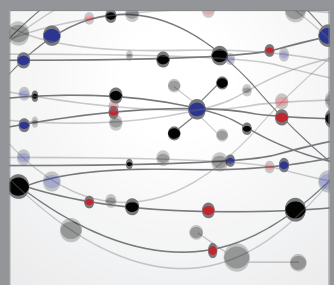

The Scientific World Journal
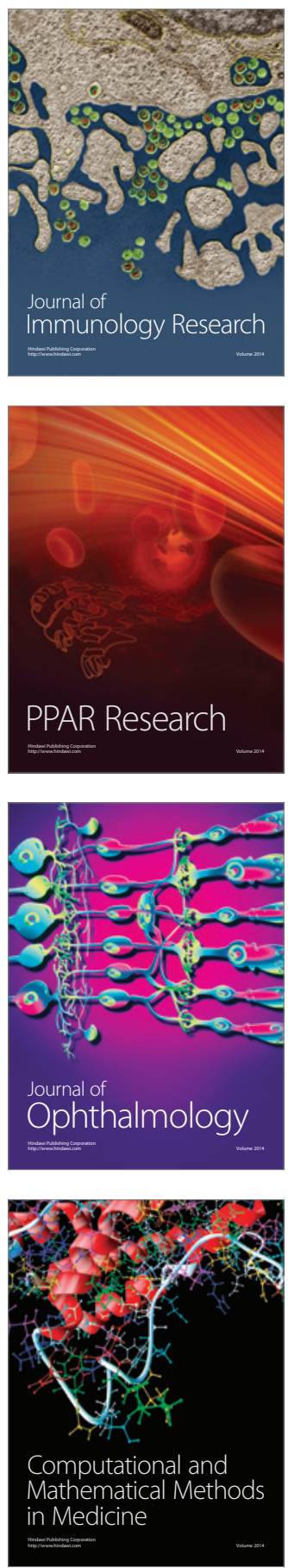

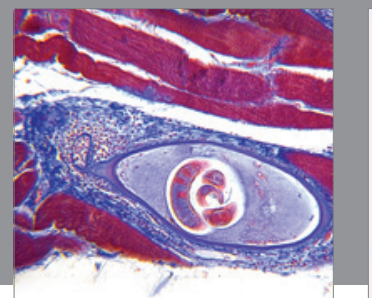

Gastroenterology

Research and Practice
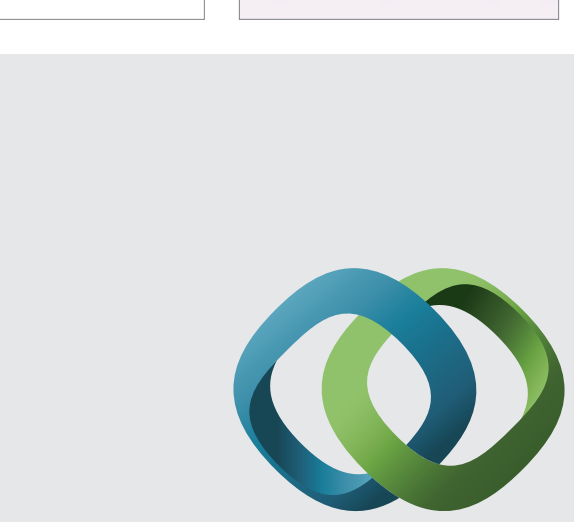

\section{Hindawi}

Submit your manuscripts at

http://www.hindawi.com
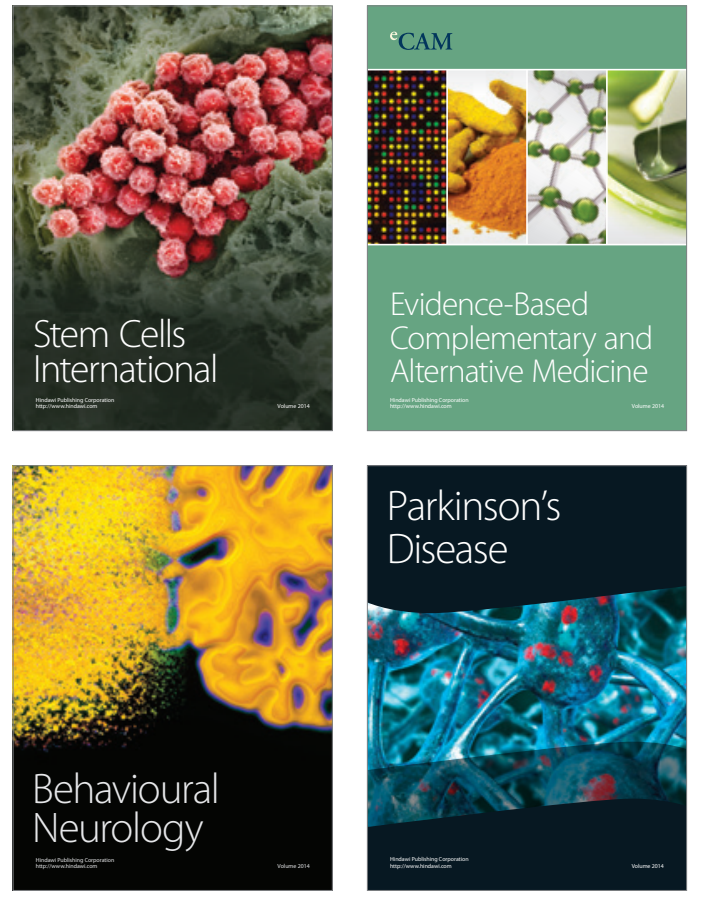
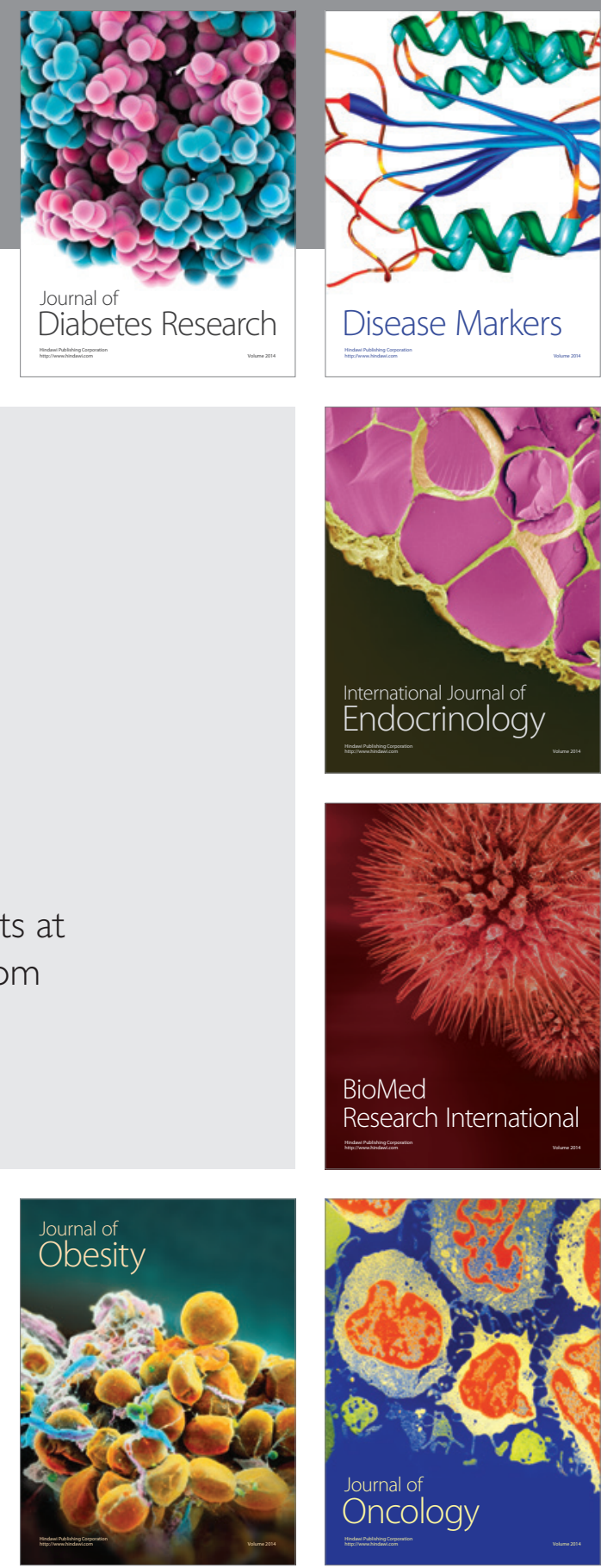

Disease Markers
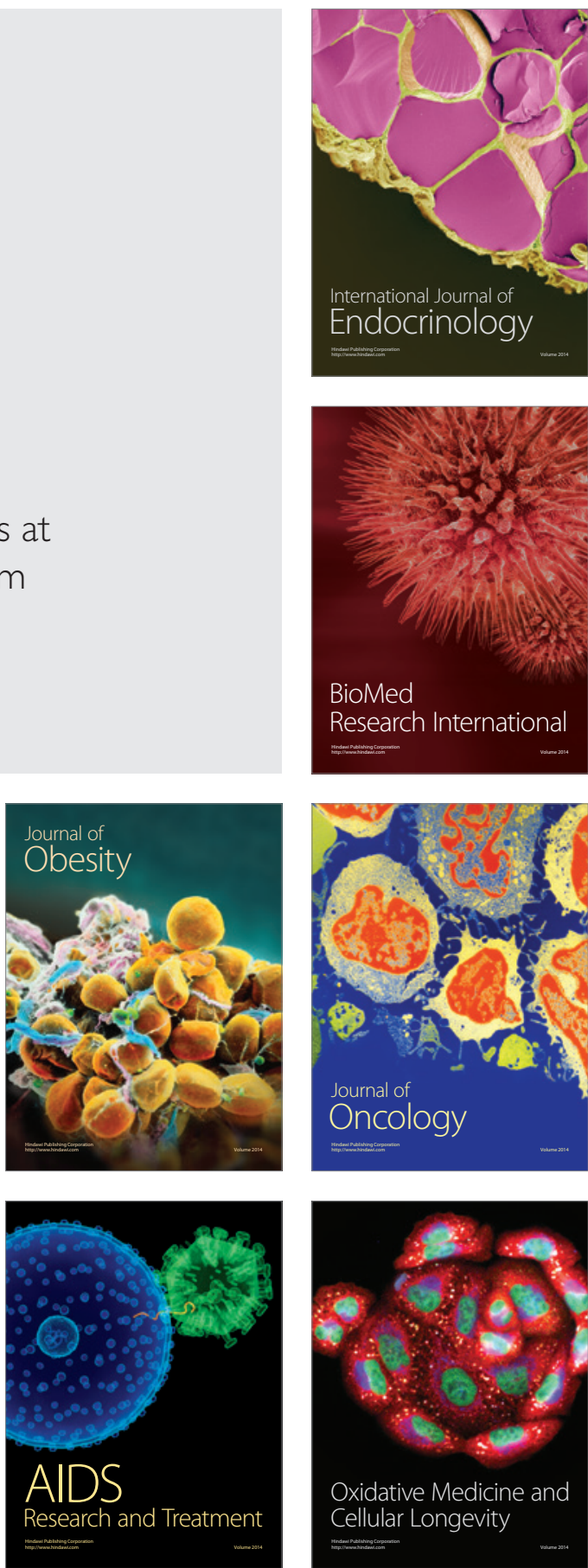